\title{
Chikungunya: treatments, opportunities and possibilities
}
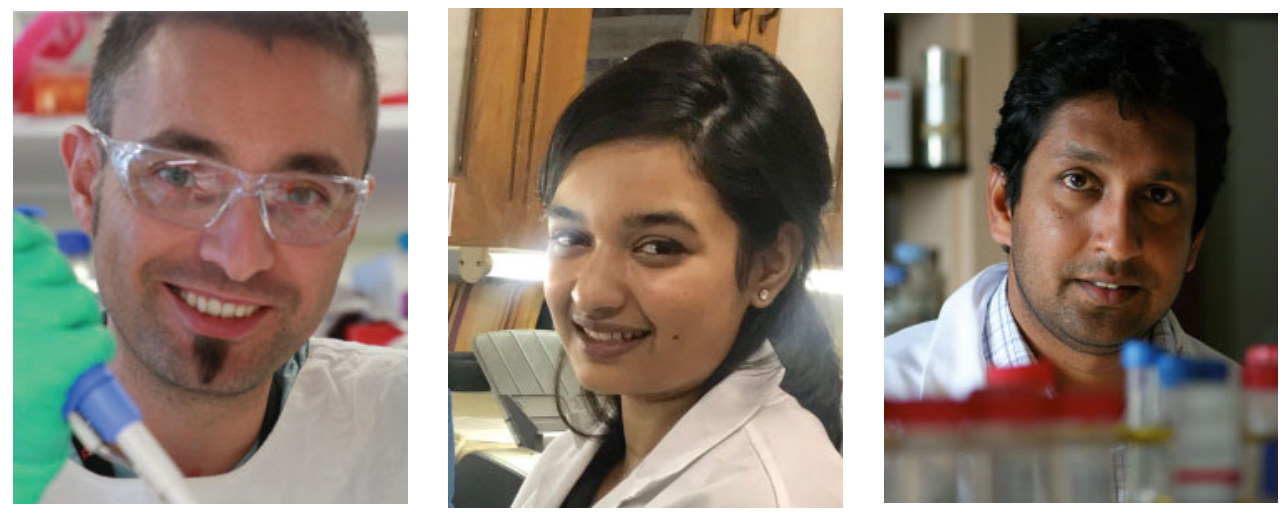

Joseph R Freitas ${ }^{\mathrm{A}, \mathrm{C}}$, Shambhavi Rao ${ }^{\mathrm{B}}$ and Suresh Mabalingam ${ }^{\mathrm{A}, \mathrm{D}}$

${ }^{\mathrm{A}}$ Institute for Glycomics, Griffith University (Gold Coast campus), Qld, Australia.

${ }^{\mathrm{B}}$ National Institute of Virology, Pune, India. Tel: +91 8826472536, Email: shambhavi2536@gmail.com

${ }^{\mathrm{C}}$ Tel: +6175552 9351, Email: j.freitas@griffith.edu.au

D Tel: +61 75552 7178, Email: s.mahalingam@griffith.edu.au

The natural progression of chikungunya virus (CHIKV) disease can consist of three stages - acute, post-acute and chronic, each having different clinical features. The acute phase (up to 3 weeks) is characterised by high viremia, fever, rash, polyarthralgia, synovitis and intense inflammation. Complete recovery is achieved in most symptomatic cases after this phase. However, in a large proportion of patients symptoms persist into a post-acute phase and in some may even continue to become chronic. In the postacute phase, which can last up to 4 months, there is clinical persistence of joint inflammation or relapse after transient improvement. These can lead to musculoskeletal disorders and eventually chronicity of disease. The main symptoms being chronic inflammatory rheumatism that can last for several years in some cases. With the near global reach, debilitating nature and recent outbreaks of CHIKV there has been much research effort put towards combatting it. New antivirals and medications to counteract inflammation are being developed. Development of CHIKV vaccines is also an area with intense research focus.

Chikungunya virus is a member of the Togaviridae family, and belongs to the Alphavirus genus. Its name is translated from the African dialect of Makonde which means, "that which bends up, and refers to the effect of the incapacitating arthralgia experienced by the majority of patients with CHIKV fever. This arthralgia affects the small joints of the hands, wrists, ankles, and feet. The arthritislike symptoms are accompanied by other symptoms such as maculopapular rash, myalgia, nausea, headaches, nasal discharge, conjunctivitis, retrobulbar pain, photophobia, and lymphadenopathy ${ }^{1}$. In the majority of cases recovery is achieved within 10 days; however, in some people the joint pain may persist for months (even years) ${ }^{1}$.

CHIKV is transmitted to people from its natural reservoirs, which include monkeys, rodents, bats, and birds, and subsequently between people by the bite of two types of mosquitos: Aedes aegypti and Aedes albopictus ${ }^{1}$. Historically, CHIKV cases have been associated with developing countries in Africa. However, it has begun to show signs of re-emergence following decades of low activity ${ }^{2}$. Several outbreaks have occurred infecting millions of people in the Indian Ocean islands, Asia, Caribbean Islands, Pacific Islands and the Americas ${ }^{2}$. The disease has an enormous economic burden due to medical costs and decreased productivity. As there are no vaccines or antivirals for prevention and treatment of CHIKV infections, there is critical need for the development of such treatments.

\section{Antiviral and anti-inflammatory compounds}

Currently, there are no specific licensed antiviral medications for CHIKV infections and treatments are limited to the use of nonsteroidal anti-inflammatory drugs (NSAID) or corticosteroid drugs to relieve fever and joint swelling ${ }^{2}$. However, with the re-emerging 
threat of CHIKV, recent years have seen interest in the discovery and testing of novel antiviral and anti-inflammatory medications to combat CHIKV.

Chloroquine, an antimalarial drug was found to be effective against alphaviruses in vitro ${ }^{3}$. However, in a double-blinded clinical trial with infected patients during the La reunion epidemic chloroquine was found to be ineffective ${ }^{4}$. Ribavirin, an antiviral agent, in combination with interferon-alpha was found to be beneficial in inhibition of SFV and CHIKV replication in vitro ${ }^{5}$. Arbidol, licensed for the treatment of influenza and other respiratory infections, was also found to be effective in inhibiting CHIKV replication in vitro $(\mathrm{IC} 50<10 \mu \mathrm{g} / \mathrm{mL})^{6}$.

High throughput screening is a common approach to identify novel antiviral compounds. Screening of compounds resulted in identification of coumarin 30 as an effective antiviral agent in inhibiting the replication of $\mathrm{CHIKV}^{7}$. Harringtonine, a cephlotoxoid alkaloid, was identified by screening a natural compound library for inhibition of CHIKV replication ${ }^{8}$.

Varghese et $a l .{ }^{9}$ have also adapted similar screening strategies to identify several compounds that inhibited CHIKV replication in a dose-dependent manner with some showing broader antiviral activity against other alphaviruses. Compounds, such as niclosamide and nitazoxanide, were found to restrict the entry of virus, restrain both viral discharge and cell-to-cell virus transfer, and exhibit expansive anti-alphavirus function against CHIKV and other alphaviruses ${ }^{10}$. Alternate modes of action for antivirals include the inhibition of viral fusion by neutralising the acidic environment of endosomal vesicles. The small-molecule antagonist of the $\mathrm{Bcl} 2$ family of proteins, Obatoclax, demonstrated such efficacy against CHIKV as well as SINV and influenza $\mathrm{A}^{11}$. Such discoveries of antiviral activity might provide a basis for the development of new human drug therapies against CHIKV and other alphavirus infections.

Other significant research discoveries include several breakthroughs in understanding the mechanism of alphaviral infections and their interactions with host defense systems. A recent study revealed the role of inflammasomes in causing severe inflammatory disease in arboviral infection. Activation of the NLRP3 inflammasome in humans and mice following CHIKV infection was shown. Inhibition of NLRP3 activation in vivo using the inhibitor MCC950 resulted in reduced inflammation and bone loss in mice. Furthermore, this in vivo inhibition showed reduced inflammation in the closely related Ross River virus (RRV), but not for mice infected with the flavivirus West Nile virus ${ }^{12}$.

Similar approaches for the identification of anti-inflammatories of specific viral-host interactions are ongoing and offer hope for the effective treatment of CHIKV. For example, the glycan derivative drug Pentosan polysulfate (PPS), that has been used to treat cystitis in the US, was shown to decrease the level of joint swelling, cartilage damage and inflammatory proteins in both CHIKV and RRV mouse models ${ }^{13}$. PPS is currently in a phase 2 clinical trial for the treatment of RRV at 4 different locations across Australia.

\section{Antibodies}

Neutralising antibodies have been found to be effective in animal models of CHIKV infection ${ }^{14,15}$. In rhesus monkeys, administration of monoclonal antibody (mAb), SVIR001 that mimics the human anti-CHIKV mAb 4N12, resulted in rapid clearance of CHIKV infection $^{15}$. A combination therapy with CTLA4-Ig (abatacept) and 4N12 antibody decreased the periarticular swelling and joint inflammation even after administration several days after infection ${ }^{16}$. Neutralising antibodies could be used prophylactically for individuals with high risk of infection such as pregnant women and persons with underlying disabilities such as diabetes and cardiovascular diseases.

\section{Vaccines}

At present there is no licensed vaccine for prevention of CHIKV infections. Much research effort is being directed in the development of an effective vaccine with studies ranging from the preclinical stage through to phase 2 trials. A common approach to developing vaccines is often through the use of an attenuated variant of the virus. A phase 2 clinical trial utilising a live, attenuated virus showed very promising results for short-term immunity with 98\% of participants being immune after 28 days. However, this fell to $85 \%$ at the one-year follow up stage ${ }^{17}$. Side-effects were reported in a small percentage of patients, with some people reporting joint pain for a short period of time.

Mutation of the nucleolar localisation sequence (NoLS) in the $\mathrm{N}$-terminal region of CHIKV capsid protein (C protein) is another example demonstrating potential as a live attenuated vaccine candidate. Mice infected with CHIKV-NoLS did not exhibit any signs of disease, had reduced viremia and proinflammatory cytokines when compared to wild type CHIKV. When the mice were challenged with CHIKV-WT at 30 days post immunisation, no disease signs and no detectable viremia were observed ${ }^{18}$.

One particular concern with the use of live attenuated viral vaccines is the potential of the virus to revert back to its native wild-type phenotype through random mutation. The vaccine tested in the phase 2 trial was attenuated due to two point mutations in the E2 glycoprotein region ${ }^{19}$. Such small changes in the viral genome, although targeted, also make the possibility of reversion high. This would not be a concern for the NoLS vaccine candidate because a substantial number of changes have been made within $\mathrm{C}$ protein 
that affects a number of its functions. This thereby makes the likelihood of reversion extremely low. Indeed, in vitro data shows a high level of stability even after multiple passages.

Alternative attenuation strategies have also been examined for the development of a CHIKV vaccine. Replacement of the subgenomic promoter of CHIKV with the internal ribosome entry site of encephalomyocarditis virus resulted in a highly attenuated, immunogenic vaccine candidate with good efficacy in mouse models. In addition, the virus was unable to replicate within mosquito cells in vivo ${ }^{20}$. Deletion of a large segment of the nsP3 gene or the entire $6 \mathrm{~K}$ gene and subsequent administration of viral particles or infectious DNA genomes proved efficacious in animal models ${ }^{21}$.

The external envelope proteins, E1 and E2, which form heterodimers on the virion's surface and are involved in CHIKV attachment and entry are also prime targets for treatment strategies. In a study involving both cell-based and murine models the N218 of CHIKV E2 protein was found to be a potent neutralising epitope. CHIKV attachment to cells was completely blocked when the IgM $3 \mathrm{E} 7 \mathrm{~b}$ antibody was used in a pre-binding neutralisation assay by binding to the surface-accessible E2-N218 residue. Prophylactic administration of $3 \mathrm{E} 7 \mathrm{~b}$ to neonate mice markedly reduced viremia and protected against CHIKV pathogenesis in various tissues. Given therapeutically at $4 \mathrm{~h}$ post-infection, 3E7b conferred a 100\% survival rate and similarly reduced CHIKV load in many tissues. These findings demonstrate the importance of the $\mathrm{E}$ proteins in the possible future development of an epitope-based vaccine ${ }^{22}$.

The use of virus-like particles (VLPs) is another strategy that has managed to pass phase 1 clinical trials ${ }^{23}$. VLPs are multi-protein structures that mimic native viral proteins often found on the outside of the virus. However, they lack the viral genome and are thus non-infectious and unable to replicate within the human recipient. This approach holds the promise of potentially yielding safer and cheaper vaccine candidates. In the phase 1 trial, neutralising antibodies were detected in all 25 adult volunteers after the second vaccination. The antibodies remained detectable even 6 months after the third vaccination. The vaccination proved to be safe and well tolerated, without serious adverse reactions ${ }^{23}$. This trial represents an important milestone in the development of a vaccine to combat this pathogen, although further studies are needed in larger and more diverse cohorts and a phase 2 trial has been planned ${ }^{24}$.

Non-replicating viral vectors are another approach to developing vaccine candidates for CHIKV. Modified Vaccinia Ankara (MVA) is a highly attenuated, avirulent poxvirus vector engineered to be unable to productively grow in human cells. MVA demonstrates a high level of antigen gene expression, even in cells that are not susceptible to infection. Investigations using MVA vaccines in animal models, has proven that it is immunogenic and protective against various infectious agents ${ }^{25}$. Several pre-clinical studies have evaluated the efficacy of MVA expressing various CHIKV structural proteins such as $\mathrm{C}, \mathrm{E} 3, \mathrm{E} 2,6 \mathrm{~K}$, and $\mathrm{E} 1$, either alone or in combination. Positive results were obtained, with the candidates eliciting neutralising antibodies in animal models and some providing protection against challenge with $\mathrm{CHIKV}^{26,27}$.

Nucleic acid based vaccines have been tested as vaccine candidates with varying success. Although proven to be safe in humans, achieving long lasting immunity has proven challenging for DNA vaccines, often requiring multiple doses. As a way to improve immunogenicity, the use of DNA-launched alphavirus replicon vectors shows promise. Replacement of the alphaviral structural genes with the antigen gene of interest elicited strong immune responses $^{28}$. The use of this approach in combination with protein antigen doses resulted in antigen-specific immune responses against CHIKV, particularly when used in a prime-boost strategy ${ }^{29}$.

Many pre-clinical studies investigating vaccine candidates often report efficacy in terms of neutralising antibodies; however, these may not be accepted as a correlate of protection by some regulatory agencies. The requirement of a Phase 3 trial for proof of vaccine efficacy and licensure means that the time and cost of development expand rapidly. This is compounded further by the fact that epidemics/outbreaks are sporadic and hard to predict. Significant numbers of trial participants in endemic countries would also need to be screened since a large proportion of people would have preexisting CHIKV antibodies. In the absence of a Phase 3 trial it may be possible to perform a comprehensive analysis of a vaccine using animal models instead, e.g. macaques, to accelerate the vaccine to market. Alternatively, a strictly controlled Phase 3 'challenge' trial model could be employed whereby vaccinated individuals are challenged at a set time point post vaccination. This could be a controversial approach but concerns could be allayed through the use of viral strain in the challenge that is not linked to causing any form of arthralgia.

CHIKV has a major economic impact on affected nations. The huge populations of India (1.3 billion) and South American countries (400 million) means well over a billion people are vulnerable to future CHIKV outbreaks. Add to this the continuing changes in global climates that could further expand the habitat of A. albopictus across the globe. This creates opportunities for future outbreaks to occur in countries not usually associated with CHIKV. It is clear that development of new therapeutics and solutions to regulatory challenges will depend on innovative thinking and collaborations between developers, laboratory personnel, regulators, funders and governments. 


\section{References}

1. Burt, F.J. et al. (2012) Chikungunya: a re-emerging virus. Lancet 379, 662-671. doi:10.1016/S0140-6736(11)60281-X

2. Burt, F.J. et al. (2017) Chikungunya virus: an update on the biology and pathogenesis of this emerging pathogen. Lancet Infect. Dis. 17, e107-e117. doi:10.1016/S1473-3099(16)30385-1

3. Helenius, A. et al. (1982) Inhibition of Semliki forest virus penetration by lysosomotropic weak bases. J. Gen. Virol. 58, 47-61. doi:10.1099/0022-131758-1-47

4. de Lamballerie, X. et al. (2009) Antiviral treatment of chikungunya virus infection. Infect. Disord. Drug Targets 9, 101-104. doi:10.2174/187152609787847712

5. Briolant, S. et al. (2004) In vitro inhibition of Chikungunya and Semliki Forest viruses replication by antiviral compounds: synergistic effect of interferon-alpha and ribavirin combination. Antiviral Res. 61, 111-117. doi:10.1016/j.antiviral. 2003.09.005

6. Delogu, I. et al. (2011) In vitro antiviral activity of arbidol against Chikungunya virus and characteristics of a selected resistant mutant. Antiviral Res. 90,99-107. doi:10.1016/j.antiviral.2011.03.182

7. Pohjala, L. et al. (2011) Inhibitors of alphavirus entry and replication identified with a stable Chikungunya replicon cell line and virus-based assavs. PLoS One $\mathbf{6}$ e28923. doi:10.1371/journal.pone.0028923

8. Kaur, P. et al. (2013) Inhibition of chikungunya virus replication by harringtonine, a novel antiviral that suppresses viral protein expression. Antimicrob. Agents Chemother. 57, 155-167. doi:10.1128/AAC.01467-12

9. Varghese, F.S. et al. (2016) Discovery of berberine, abamectin and ivermectin as antivirals against chikungunya and other alphaviruses. Antiviral Res. 126 117-124. doi:10.1016/j.antiviral.2015.12.012

10. Wang, Y.M. et al. (2016) Antiviral activities of niclosamide and nitazoxanide against chikungunya virus entry and transmission. Antiviral Res. 135, 81-90. doi:10.1016/j.antiviral.2016.10.003

11. Varghese, F.S. et al. (2017) Obatoclax inhibits alphavirus membrane fusion by neutralizing the acidic environment of endocytic compartments. Antimicrob. Agents Chemother. 61, e02227-16. doi:10.1128/AAC.02227-16

12. Chen, W. et al. (2017) Specific inhibition of NLRP3 in chikungunya disease reveals a role for inflammasomes in alphavirus-induced inflammation. Nat. Microbiol. 2 1435-1445. doi:10.1038/s41564-017-0015-4

13. Herrero, L.J. et al. (2015) Pentosan polysulfate: a novel glycosaminoglycan-like molecule for effective treatment of alphavirus-induced cartilage destruction and inflammatory disease. J. Virol. 89, 8063-8076. doi:10.1128/JVI.00224-15

14. Fric, J. et al. (2013) Use of human monoclonal antibodies to treat Chikungunya virus infection. J. Infect. Dis. 207, 319-322. doi:10.1093/infdis/jis674

15. Broeckel, R. et al. (2017) Therapeutic administration of a recombinant human monoclonal antibody reduces the severity of chikungunya virus disease in rhesus macaques. PLoSNegl. Trop. Dis. 11, e0005637. doi:10.1371/journal.pntd.0005637

16. Miner, J.J. et al. (2017) Therapy with CTLA4-Ig and an antiviral monoclonal antibody controls chikungunya virus arthritis. Sci. Transl. Med. 9. doi:10.1126/ scitranslmed.aah3438

17. Edelman, R. et al. (2000) Phase II safety and immunogenicity study of live chikungunya virus vaccine TSI-GSD-218. Am. J. Trop. Med. Hyg. 62, 681-685. doi:10.4269/ajtmh.2000.62.681

18. Taylor, A. et al. (2017) Mutation of the N-terminal region of chikungunya virus capsid protein: implications for vaccine design. MBio 8, e01970-16. doi:10.1128/ mBio.01970-16

19. Gorchakov, R. et al. (2012) Attenuation of Chikungunya virus vaccine strain 181 clone 25 is determined by two amino acid substitutions in the E2 envelope glycoprotein. J. Virol. 86, 6084-6096. doi:10.1128/JVI.06449-11

20. Plante, K. et al. (2011) Novel chikungunya vaccine candidate with an IRES-based attenuation and host range alteration mechanism. PLoS Pathog. 7, e1002142. doi:10.1371/journal.ppat.1002142

21. Hallengärd, D. et al. (2014) Novel attenuated Chikungunya vaccine candidates elicit protective immunity in C57BL/6 mice. J. Virol. 88, 2858-2866. doi:10.1128/ JVI.03453-13
22. Lam, S. et al. (2015) A potent neutralizing IgM mAb targeting the N218 epitope on E2 protein protects against Chikungunya virus pathogenesis. MAbs 7, 1178-1194. doi:10.1080/19420862.2015.1083664

23. Chang, L.J. et al. VRC 311 Study Team. (2014) Safety and tolerability of chikungunya virus-like particle vaccine in healthy adults: a phase 1 dose-escalation trial Lancet 384, 2046-2052. doi:10.1016/S0140-6736(14)61185-5

24. NIH (2015) NIH-sponsored clinical trial of chikungunya vaccine opens. https:// www.niaid.nih.gov/news-events/nih-sponsored-clinical-trial-chikungunya-vaccine-opens

25. Sutter, G. and Staib, C. (2003) Vaccinia vectors as candidate vaccines: the development of modified vaccinia virus Ankara for antigen delivery. Curr. Drug Targets Infect. Disord. 3, 263-271. doi:10.2174/1568005033481123

26. van den Doel, P. et al. (2014) Recombinant modified vaccinia virus Ankara expressing glycoprotein E2 of Chikungunya virus protects AG129 mice against lethal challenge. PLoS Negl. Trop. Dis. 8, e3101. doi:10.1371/journal.pntd. 0003101

27. Roques, P. et al. (2017) Attenuated and vectored vaccines protect nonhuman primates against Chikungunya virus. JCI Insight 2, e83527. doi:10.1172/jci. insight. 83527

28. Knudsen, M.L. et al. (2015) Alphavirus replicon DNA expressing HIV antigens is an excellent prime for boosting with recombinant modified vaccinia Ankara (MVA) or with HIV gp140 protein antigen. PLoS One 10, e0117042. doi:10.1371/journal. pone. 0117042

29. Hallengärd, D. et al. (2014) Prime-boost immunization strategies against Chikungunya virus. J. Virol. 88, 13333-13343. doi:10.1128/JVI.01926-14

\section{Biographies}

Joseph Freitas obtained his BSc honours from Murdoch University and worked as a research assistant at the Telethon Institute for Child Health Research, Perth. He is currently a research assistant and laboratory manager in the Emerging Viruses and Inflammation Research Group at the Institute for Glycomics, Griffith University. He is interested in understanding the immune responses against viral infections, with a particular focus on mosquito-borne viruses such as Ross River virus, Chikungunya virus, Zika virus and Dengue virus.

Shambhavi Rao completed her Masters in Virology from Amity University, India and bachelors' degree from Delhi University, India. Her dissertation was on the screening and characterisation of monoclonal antibodies to Zika virus, which was carried out at the National Institute of Virology, India. She is interested in studying emerging arboviruses with emphasis on biosafety, biosecurity, diagnosis and epidemiology.

Suresh Mahalingam is a viral immunologist, with a particular interest in the pathogenesis and treatment of viral-induced inflammatory diseases. He is presently an NHMRC Senior Research Fellow and Professor at the Institute for Glycomics, Griffith University in Australia. He has made numerous advances in our understanding of viral inflammatory diseases. His contribution to fundamental research is complemented by clinical and translational 'spin-offs' supported by highly productive collaborations with clinician-scientists and commercial partners. 